\title{
Visual Outcomes and Prognostic Factors of Large Submacular Hemorrhages Secondary to Polypoidal Choroidal Vasculopathy
}

\author{
Yu Matsuo' \\ Masatoshi Haruta (DD ${ }^{\prime}$ \\ Yumi Ishibashi' \\ Koki Ishibashi' \\ Kei Furushima' \\ Nobuhiro Kato' \\ Kenta Murotani ${ }^{2}$ \\ Shigeo Yoshida' \\ 'Department of Ophthalmology, Kurume \\ University School of Medicine, Kurume, \\ Japan; ${ }^{2}$ Biostatistics Center, Kurume \\ University, Kurume, Japan
}

Correspondence: Masatoshi Haruta Department of Ophthalmology, Kurume University School of Medicine, 67 Asahimachi, Kurume, Fukuoka, 830-00II, Japan Tel +8I 942317574

Fax +8I 942370324

Email haruta_masatoshi@med.kurume-u. ac.jp
Purpose: Patients with polypoidal choroidal vasculopathy (PCV) may develop large submacular hemorrhages (SMHs), which may result in severe visual loss. This study was performed to determine the visual outcomes and prognostic factors of large SMHs secondary to PCV.

Patients and Methods: We retrospectively reviewed the medical records of patients diagnosed with PCV who developed a large SMH. The best-corrected visual acuity (BCVA) data were collected at the SMH development, 1 month, 1 year after the SMH development, and at the final visit. Patients' medical information also were collected and included age, gender, systemic hypertension, current regular use of an anticoagulant or antiplatelet medication, the initial area of the $\mathrm{SMH}$, breakthrough vitreous hemorrhage, ocular treatment, and fellow eye status. Univariate and multiple regression analyses were performed to determine the prognostic factors for the BCVA 1 year after the development of large SMHs.

Results: Thirty eyes of 29 patients were included in this study. The mean area of the SMHs at the development was 17.0 disc areas. The mean follow-up period after the development of SMHs was 53.5 months. The mean BCVA at the development, 1 month, and 1 year after the development, and at the final visit were $20 / 151,20 / 263,20 / 138$, and 20/152, respectively. Multiple regression analyses indicated that a SMH 20 disc areas or larger was a significant negative factor, and the BCVA 1 month after the development was a significant positive factor affecting the BCVA 1 year after the development of large SMHs.

Conclusion: The increase in the initial area of SMH was correlated inversely with the BCVA 1 year after the development of SMH. The BCVA 1 month after the development may predict the BCVA 1 year after the development of a large SMH.

Keywords: anti-vascular endothelial growth factor therapy, indocyanine green angiography, multiple regression analyses, optical coherence tomography, pneumatic displacement

\section{Introduction}

Polypoidal choroidal vasculopathy (PCV) is a subtype of neovascular age-related macular degeneration (nAMD) that is characterized by the presence of aneurysmal polypoidal lesions with a branching vascular network. ${ }^{1}$ Up to $65 \%$ of nAMD cases in Asia have been estimated to be the PCV subtype, with a study suggesting that $54.7 \%$ of Japanese patients with presumed nAMD have PCV. ${ }^{1-4}$ The current main treatments for PCV include the use of intravitreal anti-vascular endothelial growth factor (VEGF) monotherapy or in combination with verteporfin photodynamic 
therapy (vPDT). ${ }^{1}$ The PLANET study showed that aflibercept (Eylea, Regeneron Pharmaceuticals, Tarrytown, New York, USA) monotherapy was noninferior to aflibercept therapy with rescue vPDT in patients with $P C V{ }^{2}$ In contrast, the EVEREST II study found that ranibizumab (Lucentis, Genentech Inc., South San Francisco, CA, USA) in combination with vPDT was more efficacious than ranibizumab monotherapy for achieving higher polypoidal lesion regression rates and superior visual acuity (VA) outcomes in patients with $\mathrm{PCV}^{3}$ However, patients with PCV may develop large submacular hemorrhages (SMHs) during the treatment or follow-up period, which may result in severe visual loss. ${ }^{5}$ The purpose of our study was to determine the visual outcomes in patients with PCV who developed large SMHs. We also analyzed the prognostic factors that may have affected the visual outcomes.

\section{Patients and Methods}

We retrospectively reviewed the medical records of patients with a diagnosis of PCV who presented to the macular degeneration unit of Kurume University Hospital from January 2010 to January 2021. Patients were included in this study if they presented with a $\mathrm{SMH}$ equal to or larger than 4 disc areas and if they continued to be followed at least 1 year after the development of a large SMH. This study adhered to the tenets of the Declaration of Helsinki. The Ethical Committee of Kurume University approved this study and waived the need for individual patient consent due to the retrospective nature of this study (No. 16221). The data were appropriately anonymized to protect confidentiality during the analyses.

The diagnosis of PCV was confirmed using color fundus photography (TRC-50DX, TOPCON, Tokyo, Japan), spectral-domain optical coherence tomography (OCT) (HRA+OCT Spectralis, Heidelberg Engineering, Heidelberg, Germany), and if available, fluorescein angiography (FA) and indocyanine green angiography (ICGA) (HRA+OCT Spectralis, Heidelberg Engineering, Heidelberg, Germany) (Figure 1). The best-corrected visual acuity (BCVA) data were collected from the visit before the development of the $\mathrm{SMH}$ if available, at the development, 1 month, and 1 year after the development, and at the final visit. The BCVAs were measured as decimal values and converted to the logarithm of the minimum angle of resolution ( $\log$ MAR) units for statistical analyses; further, counting fingers was graded as $\log$ MAR 1.85, hand motions as $\log$ MAR 2.3, light

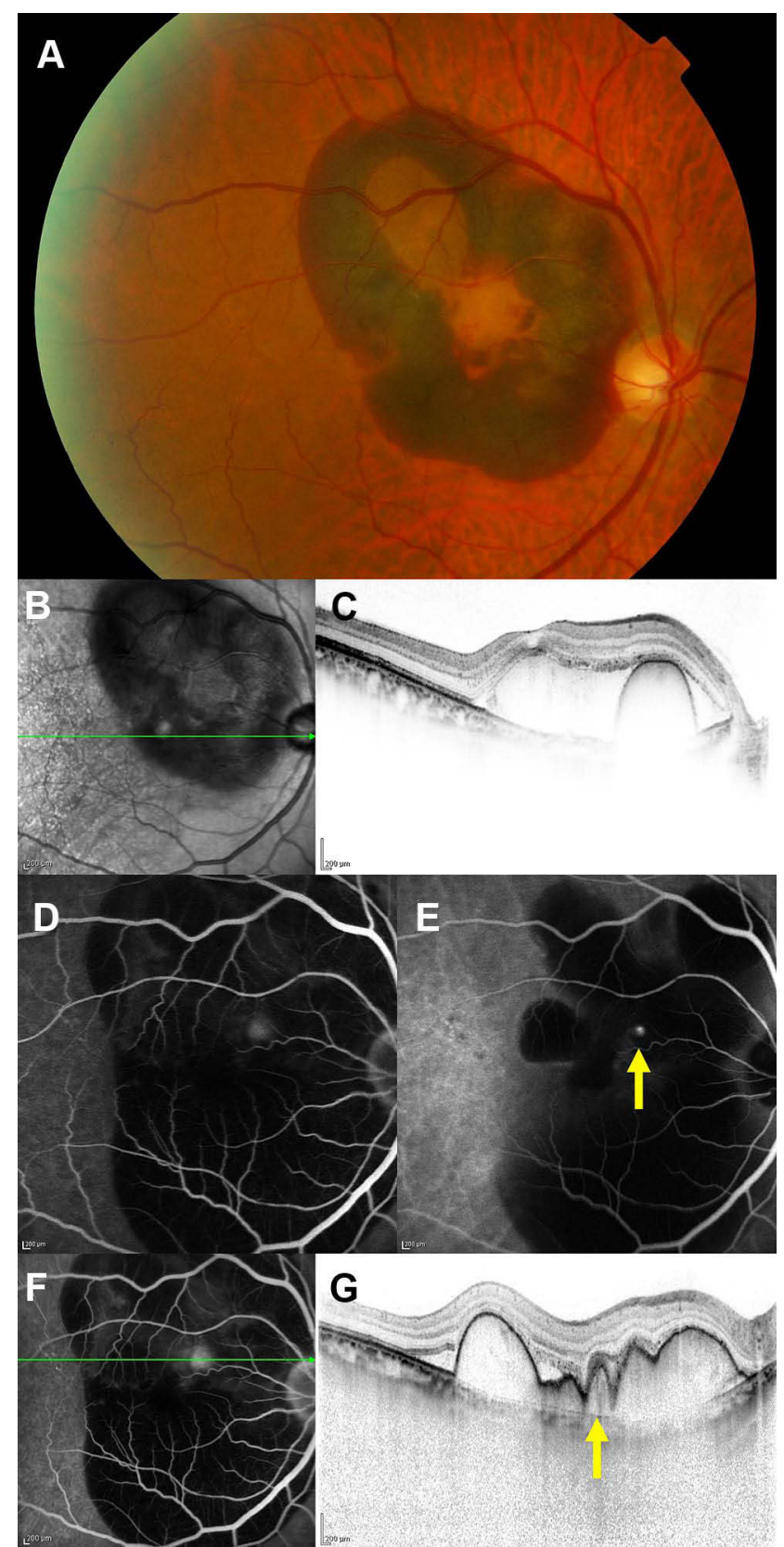

Figure I A representative case of a large submacular hemorrhage (SMH) secondary to polypoidal choroidal vasculopathy. (A) A color fundus photograph shows a large SMH. (B and C) Infrared reflectance and optical coherence tomography (OCT) images show the SMH and hemorrhagic retinal pigment epithelial detachment. (B) The green arrow indicates the direction of OCT scan in (C). ( $\mathbf{D}$ and $\mathbf{E}$ ) Fluorescein and indocyanine green angiography performed after pneumatic displacement. (E) The yellow arrow indicates a polypoidal lesion. (F and $\mathbf{G})$ Fluorescein angiography and OCT images performed after pneumatic displacement. (F) The green arrow indicates the direction of the OCT scan in $(\mathbf{G})$. (G) The yellow arrow indicates the polypoidal lesion.

perception as $\operatorname{logMAR} 2.8$, and no light perception as $\log$ MAR 2.9. Data are expressed as the mean \pm standard deviation. Patients' medical information also were collected and included patient age, gender, systemic hypertension, current regular use of an anticoagulant or 
antiplatelet medication, the initial area of the SMH, breakthrough vitreous hemorrhage, ocular treatment, and fellow eye status. The area of the SMH was measured on the fundus photographs obtained at the development of SMH. The area of the SMH was regarded as 20 disc areas when fundus photographs could not cover the entire SMH. Univariate analyses were first used to determine the potential prognostic factors for the BCVA 1 year after the development of large SMHs. Multiple regression analyses were performed using factors found to be significant in the univariate analyses. All analyses were performed using SAS version 9.4 (SAS Institute Inc., Cary, NC, USA). A $p<0.05$ was considered significant.

\section{Results}

Thirty eyes of 29 patients (24 men, 5 women) with a diagnosis of PCV who developed a SMH equal to or larger than 4 disc areas were included in this study. The clinical characteristics of the 29 patients are shown in Table 1. The mean area of the large SMHs at the development was $17.0 \pm$ 4.8 disc areas, and 11 eyes had a breakthrough vitreous hemorrhage during the follow-up period. Twenty-one eyes underwent FA and ICGA after the development of large SMHs and three eyes before the development of large SMHs. Twenty-six eyes had not been treated previously for

Table I Characteristics of Patients with Large SMHs Secondary to PCV

\begin{tabular}{|l|c|}
\hline \multicolumn{2}{|l|}{ Characteristics } \\
\hline No. eyes & 30 \\
Age (years) & $70.8 \pm 11.5$ \\
No. women (\%) & $5(17 \%)$ \\
Systemic hypertension - no. (\%) & $20(67 \%)$ \\
Anticoagulant therapy - no. (\%) & $2(7 \%)$ \\
Antiplatelet therapy - no. (\%) & $6(20 \%)$ \\
\hline Initial area of SMH & \\
20 disc areas or larger - no. (\%) & $11(37 \%)$ \\
\hline Breakthrough vitreous hemorrhage - no. (\%) & $11(37 \%)$ \\
\hline Treatment for PCV before SMH - no. (\%) & $4(13 \%)$ \\
\hline Treatment after SMH & \\
Pneumatic displacement - no. (\%) & $13(43 \%)$ \\
Anti-VEGF therapy - no. (\%) & $22(73 \%)$ \\
Pars plana vitrectomy - no. (\%) & $4(13 \%)$ \\
\hline nAMD in fellow eyes - no. (\%) & $9(30 \%)$ \\
\hline
\end{tabular}

Abbreviations: nAMD, neovascular age-related macular degeneration; PCV, polypoidal choroidal vasculopathy; SMH, submacular hemorrhage; VEGF, vascular endothelial growth factor.
PCV. Four eyes had been treated previously, ie, with intravitreal ranibizumab injections ( 3 eyes), intravitreal aflibercept injections (3 eyes), and/or vPDT (1 eye) for PCV. After the large SMHs developed, 13 eyes underwent pneumatic displacement, 22 eyes received anti-VEGF therapy, and four eyes underwent pars plana vitrectomy, ie, 2 eyes for a breakthrough vitreous hemorrhage, 1 eye for a macular hole retinal detachment and breakthrough vitreous hemorrhage, and 1 eye for a rhegmatogenous retinal detachment. The mean age of the study patients was $70.8 \pm 11.5$ years. The mean follow-up period after the development of SMHs was $53.5 \pm 30.3$ months.

The mean BCVA at the development, 1 month, and 1 year after the development of large SMHs, and at the final visit were $\log$ MAR $0.88 \pm 0.61$ (Snellen equivalent 20/151), logMAR $1.12 \pm 0.82$ (Snellen equivalent 20/263), $\log$ MAR $0.84 \pm 0.72$ (Snellen equivalent 20/138), and logMAR $0.88 \pm 0.75$ (Snellen equivalent 20/152), respectively (Figure 2). Subgroup analyses of nine eyes in which the BCVA was measured before the development of large SMHs showed that the mean BCVA before the development, at the development, 1 month, and 1 year after the development of large SMHs, and at the final visit were logMAR $0.23 \pm 0.14$ (Snellen equivalent 20/34), $\log$ MAR $0.74 \pm 0.44$ (Snellen equivalent 20/111), logMAR $1.26 \pm 0.77$ (Snellen equivalent 20/360), $\log$ MAR $1.08 \pm 0.43$ (Snellen equivalent 20/239), and $\log$ MAR $1.22 \pm 0.47$ (Snellen equivalent 20/ $333)$, respectively.

The prognostic factors evaluated as predictive of the BCVA 1 year after the development of large SMHs are shown in Table 2. Univariate analyses showed that the presence of systemic hypertension, the initial area of the $\mathrm{SMH}$ equal to or larger than 20 disc areas, the presence of breakthrough vitreous hemorrhage, pneumatic displacement, anti-VEGF therapy after the development of large SMHs, and the BCVAs 1 month after the development were significant factors affecting the BCVA 1 year after the development of large SMHs. Multiple regression analyses indicated that the initial area of the SMH equal to or larger than 20 disc areas was a significant negative factor, and the BCVA 1 month after the development was a significant positive factor affecting the BCVA 1 year after the development of large SMHs (Table 2).

\section{Discussion}

The natural history of SMHs associated with nAMD has been reported to result in poor visual outcomes. Avery et al showed that progressive loss of VA from baseline occurred 


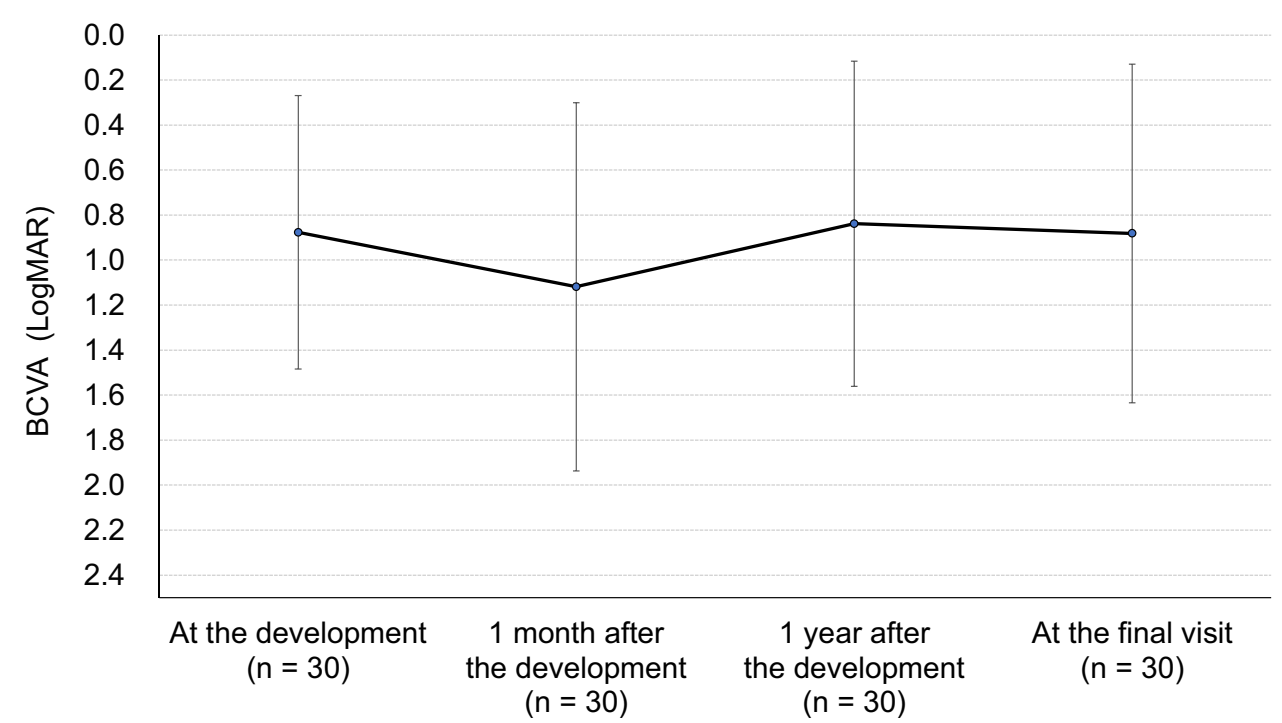

Figure 2 The best-corrected visual acuities (BCVAs) expressed in logarithm of the minimum angle of resolution (logMAR) units (mean \pm standard deviation) at the development, I month, and I year after the development of large submacular hemorrhages, and at the final visit.

in most eyes throughout a 3-year follow-up period. ${ }^{6}$ Scupola et al reported that the mean BCVA worsened from 20/240 at the development to $20 / 1250$ at the final visit during a 2-year follow-up period. ${ }^{7}$ When untreated, SMHs can damage the macular photoreceptors as the result of iron toxicity, fibrin meshwork contraction, and reduced nutrient flux, with subsequent macular scarring. ${ }^{8}$

Massive SMHs often develop in eyes with PCV and are devastating complications with irreversible visual impairment. Cho et al reported that the incidence rate of massive SMHs in eyes with PCV is $29.9 \%$ within 10 years of follow-up. ${ }^{5}$ Those authors also showed that the mean BCVA worsened from 20/71 at the development to 20/ 4381 year after the development of massive SMHs, and $71.9 \%$ of eyes had BCVAs worse than 20/200 1 year after the development. In the current study, all 30 eyes diagnosed with PCV presented with a large SMH equal to or larger than 4 disc areas. The mean BCVA improved slightly from 20/151 at the development to 20/138 1 year later; however, $30.0 \%$ of eyes had a BCVA worse than 20/ 2001 year after the development. In nine eyes in which the BCVA was measured before a large SMH developed,

Table 2 Prognostic Factors Predictive of BCVA I Year After Development of Large SMHs Secondary to PCV

\begin{tabular}{|c|c|c|c|c|c|c|}
\hline & \multicolumn{3}{|c|}{ Univariate Analyses } & \multicolumn{3}{|c|}{ Multiple Regression Analyses } \\
\hline & Coefficient & $95 \% \mathrm{Cl}$ & $\mathrm{p}$ value & Coefficient & $95 \% \mathrm{Cl}$ & p value \\
\hline Age & 0.017 & $-0.00583-0.04035$ & 0.137 & & & \\
\hline Gender (female) & 0.062 & $-0.67524-0.79924$ & 0.865 & & & \\
\hline Systemic hypertension & 0.553 & $0.00999-1.09501$ & 0.046 & 0.129 & $-0.14952-0.40701$ & 0.349 \\
\hline Anticoagulant therapy & 0.013 & $-1.08954-1.11454$ & 0.982 & & & \\
\hline Antiplatelet therapy & -0.298 & $-0.97542-0.37959$ & 0.375 & & & \\
\hline Initial area of SMH 20 disc areas or larger & 1.022 & $0.61137-1.43313$ & $<0.001$ & 0.481 & $0.16266-0.80012$ & 0.005 \\
\hline Breakthrough vitreous hemorrhage & 0.650 & $0.13804-1.16196$ & 0.015 & -0.278 & $-0.61652-0.06099$ & 0.103 \\
\hline Treatment for PCV before SMH & 0.215 & $-0.58899-1.01976$ & 0.588 & & & \\
\hline Pneumatic displacement after SMH & -0.529 & $-1.04473--0.01364$ & 0.045 & -0.243 & $-0.5|9| 9-0.0331 \mid$ & 0.082 \\
\hline Anti-VEGF therapy after SMH & -0.953 & $-1.45363--0.45319$ & $<0.001$ & -0.213 & $-0.55068-0.12421$ & 0.204 \\
\hline Pars plana vitrectomy after SMH & 0.244 & $-0.5589 \mid-1.04737$ & 0.538 & & & \\
\hline nAMD in fellow eyes & 0.183 & $-0.41233-0.779$ & 0.534 & & & \\
\hline BCVA I month after development & 0.721 & $0.5240 I-0.91825$ & $<0.001$ & 0.564 & $0.34847-0.779$ & $<0.001$ \\
\hline
\end{tabular}

Note: Figures in bold style represent $p<0.05$.

Abbreviations: $\mathrm{BCVA}$, best-corrected visual acuity; $\mathrm{Cl}$, confidence interval; $\mathrm{nAMD}$, neovascular age-related macular degeneration; PCV, polypoidal choroidal vasculopathy; SMH, submacular hemorrhage; VEGF, vascular endothelial growth factor. 
the mean BCVA worsened from 20/34 before the development to 20/239 1 year after the development.

Previous studies have shown that the long-term visual outcomes of SMHs associated with nAMD were correlated inversely with the initial size of the SMHs. Scupola et al reported that the initial sizes of the SMHs were correlated with the final VA. ${ }^{7}$ Avery et al also reported a significant relationship between the initial size of the SMHs and visual outcomes at the 12-month examination. ${ }^{6}$ In the current study, multiple regression analyses confirmed that the initial area of the SMHs equal to or larger than 20 disc areas decreased the BCVA 1 year after the development.

Multiple regression analyses also showed that the BCVA 1 month after the development was correlated with the BCVA 1 year after the development of large SMHs, which suggests that the BCVA 1 month after may predict the BCVA 1 year after the development of large SMHs. In addition, the mean BCVA at the final visit was worse than the mean BCVA 1 year after the development of SMHs. This may be related to the fact that the underlying PCV continues to progress even after the development of SMHs. Chang et al suggested that postoperative anti-VEGF therapy may be needed to maintain the visual gains obtained by treating the SMHs. ${ }^{9}$ However, Baek et al reported that the activity of the PCV lesions diminishes after the development of large SMHs, which may lead to a reduction in the frequency of anti-VEGF injections. ${ }^{10}$

Multiple treatment methods, including vitrectomy with subretinal tissue plasminogen activator (tPA), pneumatic displacement with and without intravitreal tPA, and intravitreal anti-VEGF therapy, have been used to treat SMHs secondary to nAMD. ${ }^{11}$ However, no consensus exists regarding the optimal treatment of large SMHs associated with PCV. Kang et al compared three treatment methods for SMHs secondary to PCV including intravitreal anti-VEGF monotherapy, pneumatic displacement with anti-VEGF therapy, and pars plana vitrectomy with anti-VEGF therapy, and found little difference in the VAs at 2 years among the three treatments. ${ }^{12}$ In the current study, multiple regression analyses showed that neither pneumatic displacement nor anti-VEGF therapy after the development of large SMHs significantly affected the BCVA 1 year later; however, these results may be attributable to the small sample sizes.

The current study had limitations that included its retrospective nature, the small number of cases, and no control group. The treatment methods were not standardized and differed among the patients. However, the study provided the 1-year real-world visual outcomes of large SMHs secondary to PCV with the prognostic factors evaluated by univariate and multiple regression analyses.

\section{Conclusion}

We reported the clinical characteristics and visual outcomes of 30 eyes of 29 patients with a diagnosis of PCV who developed SMHs equal to or larger than 4 disc areas. The increase in the initial area of the SMHs was correlated inversely with the BCVA 1 year after the development of large SMHs. The BCVA at 1 month after may predict the BCVA 1 year after the development of large SMHs.

\section{Acknowledgments}

The authors thank Lynda Charters for English editing.

\section{Disclosure}

Dr Yumi Ishibashi reports personal fees from Santen Pharmaceutical Co., Ltd. and Bayer Yakuhin, Ltd., outside the submitted work. Dr Koki Ishibashi reports personal fees from Novartis Japan, outside the submitted work. The authors report no other conflicts of interest associated with this work.

\section{References}

1. Cheung CMG, Lai TYY, Ruamviboonsuk P, et al. Polypoidal choroidal vasculopathy: definition, pathogenesis, diagnosis, and management. Ophthalmology. 2018;125:708-724. doi:10.1016/j.ophtha.2017.11.019

2. Wong TY, Ogura Y, Lee WK, et al. Efficacy and safety of intravitreal aflibercept for polypoidal choroidal vasculopathy: two-year results of the aflibercept in polypoidal choroidal vasculopathy study. $\mathrm{Am}$ J Ophthalmol. 2019;204:80-89. doi:10.1016/j.ajo.2019.02.027

3. Lim TH, Lai TYY, Takahashi K, et al. Comparison of ranibizumab with or without verteporfin photodynamic therapy for polypoidal choroidal vasculopathy: the EVEREST II randomized clinical trial. JAMA Ophthalmol. 2020;138:935-942. doi:10.1001/ jamaophthalmol.2020.2443

4. Maruko I, Iida T, Saito M, Nagayama D, Saito K. Clinical characteristics of exudative age-related macular degeneration in Japanese patients. Am $J$ Ophthalmol. 2007;144:15-22. doi:10.1016/j. ajo.2007.03.047

5. Cho JH, Ryoo NK, Cho KH, Park SJ, Park KH, Woo SJ. Incidence rate of massive submacular hemorrhage and its risk factors in polypoidal choroidal vasculopathy. Am $J$ Ophthalmol. 2016;169:79-88. doi:10.1016/j.ajo.2016.06.014

6. Avery RL, Fekrat S, Hawkins BS, Bressler NM. Natural history of subfoveal subretinal hemorrhage in age-related macular degeneration. Retina. 1996;16:183-189. doi:10.1097/00006982-199616030-00001

7. Scupola A, Coscas G, Soubrane G, Balestrazzi E. Natural history of macular subretinal hemorrhage in age-related macular degeneration. Ophthalmologica. 1999;213:97-102. doi:10.1159/000027400

8. Stanescu-Segall D, Balta F, Jackson TL. Submacular hemorrhage in neovascular age-related macular degeneration: a synthesis of the literature. Surv Ophthalmol. 2016;61:18-32. doi:10.1016/j. survophthal.2015.04.004 
9. Chang W, Garg SJ, Maturi R, et al. Management of thick submacular hemorrhage with subretinal tissue plasminogen activator and pneumatic displacement for age-related macular degeneration. $\mathrm{Am}$ J Ophthalmol. 2014;157:1250-1257. doi:10.1016/j.ajo.2014.02.007

10. Baek J, Kim JH, Lee MY, Lee WK. Disease activity after development of large subretinal hemorrhage in polypoidal choroidal vasculopathy. Retina. 2018;38:1993-2000. doi:10.1097/IAE.00000 00000001817
11. Shultz RW, Bakri SJ. Treatment for submacular hemorrhage associated with neovascular age-related macular degeneration. Semin Ophthalmol. 2011;26:361-371. doi:10.3109/08820538.2011.585368

12. Kang HG, Kang H, Byeon SH, et al. Long-term visual outcomes for treatment of submacular haemorrhage secondary to polypoidal choroidal vasculopathy. Clin Exp Ophthalmol. 2018;46:916-925. doi:10.1111/ceo.13198

\section{Publish your work in this journal}

Clinical Ophthalmology is an international, peer-reviewed journal covering all subspecialties within ophthalmology. Key topics include: Optometry; Visual science; Pharmacology and drug therapy in eye diseases; Basic Sciences; Primary and Secondary eye care; Patient Safety and Quality of Care Improvements. This journal is indexed on PubMed

Submit your manuscript here: https://www.dovepress.com/clinical-ophthalmology-journal
Central and CAS, and is the official journal of The Society of Clinical Ophthalmology (SCO). The manuscript management system is completely online and includes a very quick and fair peer-review system, which is all easy to use. Visit http://www.dovepress.com/ testimonials.php to read real quotes from published authors. 